\title{
HE I 5876 LINE STRUCTURE IN THE SPECTRA OF IL CEP A
}

\author{
N.Z.Ismailov, O.V.Khalilov, G.R.Bahaddinova \\ Shamakhy Astrophysical Observatory after named N.Tusi of ANAS \\ AZ 5626, Y.Mamedaliyev set., ShAO, Shamakhy, Azerbaijan, ismailovn@yahoo.com
}

\begin{abstract}
We are presents the results of researches of the He $1 \lambda 5876 \AA\left(\mathrm{D}_{3}\right)$ line in the spectrum of Herbig Be type star IL Cep A. This line represents the absorption structure which has a width on the level of the continuum about 15-18 $\AA$, and a FWHM about 6-8 $\AA$. It is shown that the line Hel $\lambda 5876 \AA$ has a complex, multi-component structure. The average value of the radial velocities showed smooth variability, and the maximum value $\mathrm{Vr}$ achieved in 2009-2010. Large scatter in radial velocity was obtained in 2013 and 2014.

The equivalent width of the line also shows significant changes from year to year, and during the observation season. The total average value of this parameter remains constant for all observations. For all of the data, a mean value of the equivalent width of the absorption is equal $0.56 \pm 0.025 \AA$, and the standard deviation $\pm 0.16 \AA$ from the mean. The observed features in the line indicates that the field of origin this line has a complex structure and physical conditions.
\end{abstract}

Keywords: Pre-Main Sequence stars, spectral variability, helium $\mathrm{D}_{3}$ line, IL Cep A.

\section{Introduction}

Herbig Ae/Be stars (HAeBes) are pre-main sequence (PMS) objects of intermediate mass approximately from 2 to

$8 \mathrm{M}_{\odot}$ (Herbig, 1960; Finkenzeller \& Mundt, 1984; Thé et al., 1994), spectral classes from B0 to F2 and types of luminosities IV-V. They are surrounded by dust/gas accretion disks. Disks of HAeBes have a complex spatial structure and contains an accretion regions and matter outflows in the form of a stellar/disk wind at higher latitudes.

$\mathrm{HAeBe}$ stars may be the precursors of young main sequence $\beta$-Pictoris and Vega-type stars. These latter systems are surrounded by circumstellar debris disks, which perhaps contain planetary bodies. This would imply that the environment around HAEBE stars represents an early phase in the formation of planets.

HAeBe stars are important astrophysical objects because they represent the late formative stages of intermediate mass stars. They are therefore significant for understanding general and specific phenomena involved in star formation. Moreover, HAeBe stars can help us to understand a number of perplexing properties of their main sequence (MS) descendants: in particular chemical peculiarities, very slow rotation, and magnetic fields, observed in individually or in combination in a significant fraction of MS A/B stars.
The star HD $216629=$ IL Cep A $\left(\alpha_{2000}=22^{\mathrm{h}} 53^{\mathrm{m}} 16^{\mathrm{s}}\right.$, $\left.\delta_{2000}=+62^{\circ} 08^{\prime \prime} 45^{\prime}, \quad V \sim 9 .{ }^{\mathrm{m}} 3\right)$ is a member of young starformation region Cep OB3. At the distance 6.9" (IL $\mathrm{CepB}=\mathrm{ADS}$ 16341B) (Pirzkal et al., 1997) and even 0.44" (Wheelwright et al., 2010) of the star it were separated two companions which are weaker in brightness to $\Delta \mathrm{B}=3.5^{\mathrm{m}}$ than the main component.

On the data of different authors the spectral type of IL CepA was determined as B2IV-V (Garmany, 1973), B3e (Finkenzeller, 1985), or as a components in visual binary B3 and B9 (Alecian et al., 2013). Radial velocities, equivalent widths and profiles of hydrogen lines are variable (see, e.g. Ismailov et al. 2013 and references there).

Hill (1967) firstly have determined the periodical variation in brightness of the star with the period $1 .{ }^{\mathrm{d}} 401$, but later in the work (Hill et al., 1976) this periodicity was not confirmed.

One of consider question in the HAeBes spectrum is a structure and origin of the line $\mathrm{D}_{3}$. This problem firstly in detail was studied by Böhm and Catala (1995). These authors showed that the line $\mathrm{D}_{3}$ in HAeBes has mainly 2 type profiles: 1) clean absorption profiles and 2) absorption profiles which have blue shifted emission components. Some stars shows more complex structure. A question of the nature of different components in the line $\mathrm{D}_{3}$ of young stars is object of discussion.

In this work we have presented results of long time researches the line $\mathrm{D}_{3}$ structure in the spectrum of IL CepA.

\section{Observations and results}

The spectra were taken in 2006-2014 in the Cassegrain focus of the $2 \mathrm{~m}$ telescope of the Shamakhy Astrophysical Observatory of the Azerbaijan National Academy of Sciences. We used the UAGS-based spectrometer (Ismailov et al., 2013). A CCD detector is fixed in the focal plane of the camera. The $530 \times 580 \mathrm{px}$ CCD has a linear size of $9.5 \times 13.9 \mathrm{~mm}^{2}$ and a pixel size of $18 \times 24 \mu \mathrm{m}^{2}$. The dispersion is equal to $10.5 \AA / \mathrm{mm}$ and about $6 \AA / \mathrm{mm}$ in the red and blue parts of the spectrum, respectively. The entire observed region of the spectrum can be covered by two frames. The spectrometer operates in 70-140 orders. The CCD window accommodates 28 and 50 orders in the red and blue parts of the spectrum, respectively. The normal spectrograph slit width determined by the CCD pixel size is $0.35 \mathrm{~mm}$, which, given the effective Cassegrain focal length of $29500 \mathrm{~mm}$, corresponds to 2 . 
The spectrometer has a spectral resolution of $\mathrm{R}=14000$, and a signal-to-noise ratio $\mathrm{S} / \mathrm{N}=80-100$ in the $\mathrm{H} \alpha$ region and $\mathrm{S} / \mathrm{N}=20-40$ in the HeI $5876 \AA$ region. To control the stability of the system, the spectra of standard stars, the flat field image, and the comparison spectrum were taken every observing night at the beginning and end of the exposure of the object. Wavelength calibration was based on the spectrum of sunlight scattered in the Earth's atmosphere. We used the DECH20T program and its components developed at the Special Astrophysical Observatory (Galazatdinov, 1992) to perform all the image reduction tasks and subsequent measurements of the spectra.

We used the lines of the Th-Ar lamp comparison spectrum to estimate the instrumental contour width, which we found to be 0.36 and $0.44 \AA$ in the blue and red parts of the spectrum, respectively. The measured half widths of some lines exceed significantly the width of the instrumental profile: they are equal to $6-8 \AA, 2-4 \AA$, and 4-7 $\AA$, for $\mathrm{H \alpha}$, $\mathrm{H} \beta$, and $\mathrm{D}_{3}$, respectively, and $0.8-1 \AA$ and $1.5-2.0 \AA$ for $\mathrm{NaI}$ $\mathrm{D}_{1}, \mathrm{D}_{2}$ and DIBs $\lambda \lambda 5780,5798 \AA$, respectively.

We acquired a total of 50 pairs of spectrograms of the star for 2006-2014 years. Each pair consists of two identical spectra taken one after another. Three to four identical spectra of the star were taken on some nights.

The errors of the measured equivalent widths and intensities were equal to $5 \%$ and about $1 \%$, respectively. The mean error of position measurements in the spectra of standard stars was $2-3 \mathrm{~km} / \mathrm{s}$. The measurement errors for the equivalent widths $\mathrm{D}_{3}$ absorption sometimes exceed this limit because of the lower $\mathrm{S} / \mathrm{N}$ ratio in the corresponding parts of the spectrum.

The line $\mathrm{D}_{3}$ in the spectrum of IL CepA is represented as a wide absorption profile with width on the continuum at $15-18 \AA$ and with a half width FWHM 6-8 $\AA$. For this star in the work Tuairisg et al. (2000) was determined equivalent width of $\mathrm{D}_{3}$ as $250 \mathrm{~m} \AA$ for absorption and 5 $\mathrm{m} \AA$ for emission components.

Because the line in our spectra has a complex structure the equivalent width we have determined by integration method for all boundary of contours. The radial velocity of the line was measured on the displacement of the line center at the half intensity level.

In the Figure 1 has shown a time variation of equivalent widths $W_{\lambda}$ (up panel) and radial velocities $V r$ for the line $\mathrm{D}_{3}$ (top panel).

As seen from the Figure 1 values of the equivalent width have some scatter from night to night but mean of this parameter is not so large changes during all time of observation. For all measurements mean value of equivalent width is equal $0.56 \AA$ with dispersion $\pm 0.16 \AA$ and with mean error of measurement $\pm 0.025 \AA$.

In 2013 radial velocities has shown variation from -12 to $-32 \mathrm{~km} / \mathrm{s}$, this scatter bigger than in previous years. In 2014 the scatter in $V r$ is maximal, from -27 to $+44 \mathrm{~km} / \mathrm{s}$. Moreover one can see smooth decrease of mean values $\mathrm{Vr}$ still 2010, then return to previous values. This character of variation in $V r$ of the line $\mathrm{D}_{3}$ in more little scale is repeated variations of radial velocity of the emission component of $\mathrm{H} \alpha$. This event also followed with unusual decreasing of equivalent width of $\mathrm{H} \alpha$ emission from $-14 \AA$ to $-7 \AA$ (Ismailov et al., 2013).
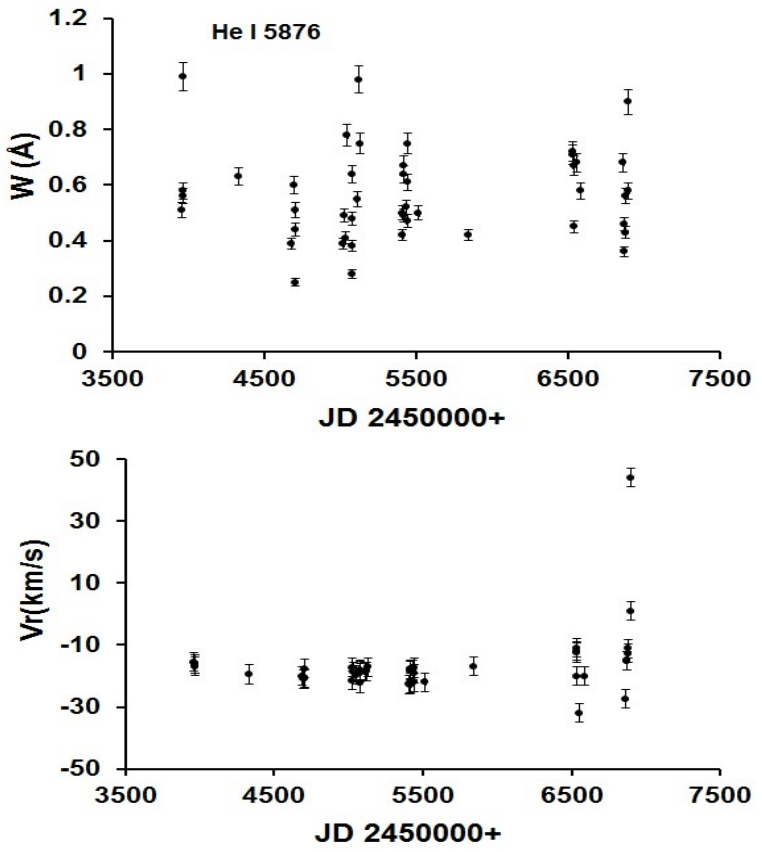

Figure 1: Time variation of equivalent widths $W_{\lambda}$ (top panel) and radial velocities $\operatorname{Vr}$ (bottom) of the line $\mathrm{D}_{3}$.

In Figure 2 are shown profiles of the line $\mathrm{D}_{3}$ obtained in various nights. The profile shows complex structure which are consisting of some components. The structure shows variations for different nights. Moreover one can see some stable observable per night components. These components at 10-20 times stronger than the noise level, because we think that they are real components, possible consisting of emission components imposed to the weak absorption. Actually as has shown in Figure 2, the limit of noise intensity must be less or equal of the intensities of atmospheric $\mathrm{H}_{2} \mathrm{O}$ lines placed near of lines D1, D2 NaI.

In detail a theory gives the following atomic transitions of the line He I $5876 \AA$ (see, http://physics.nist.gov/ PhysRefData/ASD/lines_form.html) (Table 1).

Table 1: Atomic parameters of the line $\mathrm{D}_{3}$.

\begin{tabular}{|c|c|c|c|}
\hline $\begin{array}{c}\text { Wavelength, } \\
\AA\end{array}$ & $\begin{array}{c}\text { Transition } \\
\text { level }\end{array}$ & $\log g f$ & $\begin{array}{c}\text { relative } \\
\text { intensity }\end{array}$ \\
\hline 5875.5987 & $2 \mathrm{p}^{3} \mathrm{P}^{\mathrm{o}}-3 \mathrm{~d}^{3} \mathrm{D}$ & -1.511 & \\
5875.6140 & $2 \mathrm{p}^{3} \mathrm{P}^{\mathrm{o}}-3 \mathrm{~d}^{3} \mathrm{D}$ & 0.480 & 5 \\
5875.6148 & $2 \mathrm{p}^{3} \mathrm{P}^{\mathrm{o}}-3 \mathrm{~d}^{3} \mathrm{D}$ & & 5 \\
5875.6251 & $2 \mathrm{p}{ }^{3} \mathrm{P}^{\mathrm{o}}-3 \mathrm{~d}^{3} \mathrm{D}$ & -0.338 & 5 \\
5875.6404 & $2 \mathrm{p}{ }^{3} \mathrm{P}^{\mathrm{o}}-3 \mathrm{~d}^{3} \mathrm{D}$ & 0.138 & 5 \\
5875.9663 & $2 \mathrm{p}^{3} \mathrm{P}^{\mathrm{o}}-3 \mathrm{~d}^{3} \mathrm{D}$ & -0.214 & 1 \\
\hline
\end{tabular}

In columns were presented: wavelength in $\AA$, levels of transition, $\log g f$, where $g$-factor and $f$ - the strength of oscillator, relative intensity. In our spectrum the lines 5875.61, 5875.62 and 5875.64 we have got as one unresolved line. The line 5875.966 is relative week than previous lines. Indeed as have seen in Figure 2 as a minimum we obtained 2-3 relative strong separated components in this line. But we count that these components may be results of variable emission which imposed to the wide absorption of this line. 


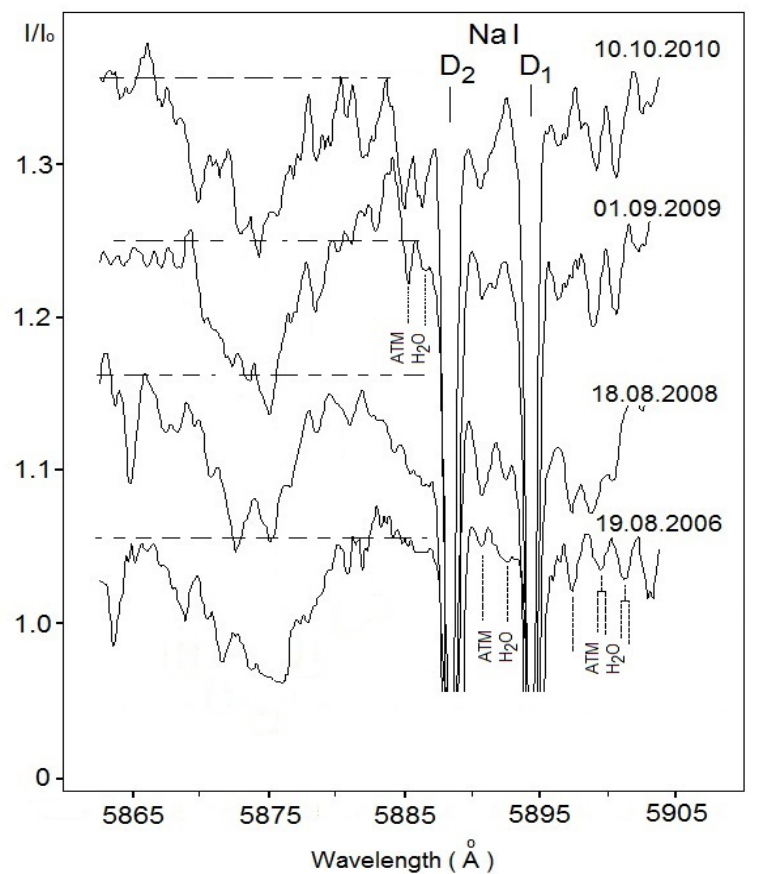

Figure 2: The structure of the line $\mathrm{D}_{3}$ in the spectrum IL CepA.
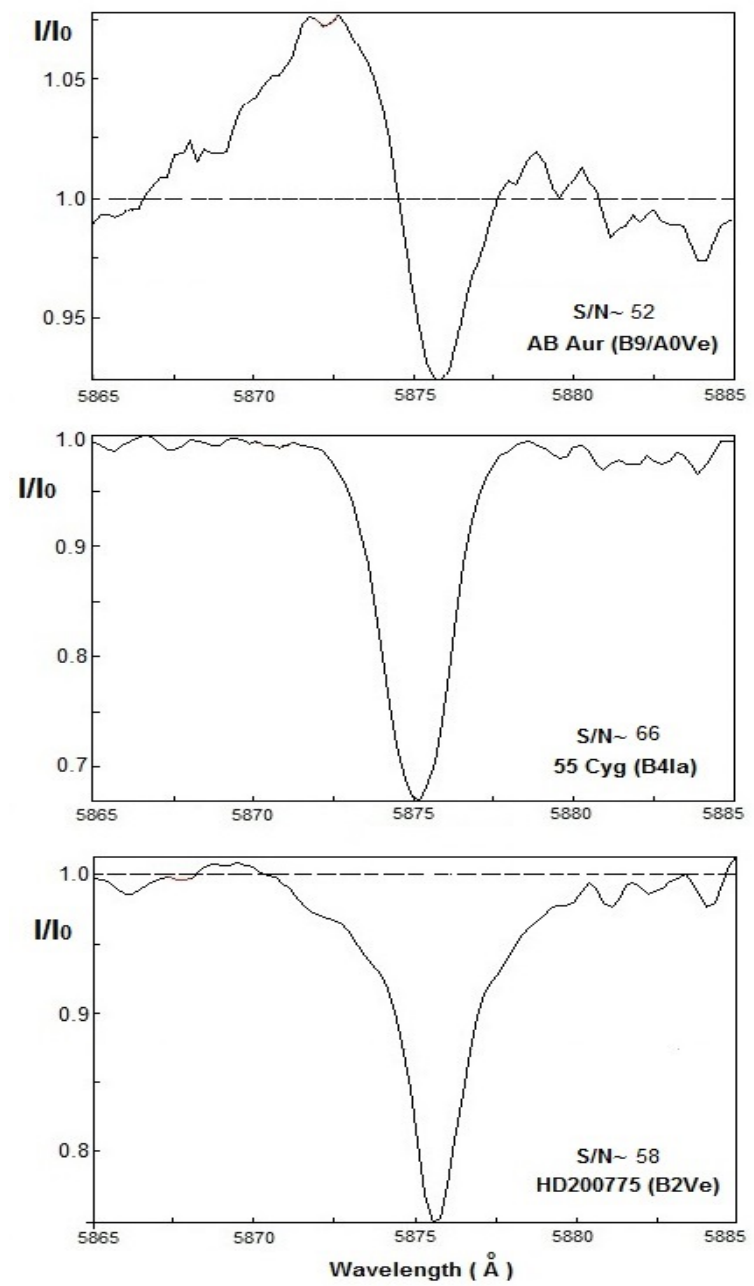

In favor of our assumptions in Figure 3 have presented the profile of the line $\mathrm{D}_{3} \mathrm{He} \mathrm{I}$ for spectrum of different HAeBes - AB Aur (Sp B9-A0V), HD 200775 (Sp B2$\mathrm{B} 3 \mathrm{~V}$ ) and supergiant $55 \mathrm{Cyg}$ (Sp B3 Ia) which were observed in the same up mentioned equipment. As seen from the Figure 2 in spite of the fact that all these stars have in the spectrum the line $\mathrm{D}_{3}$, the structure of the line as in IL Cep A is not detected. Moreover we have presented the profile of this line, for the spectrum IL Cep A obtained by Böhm and Catala (1995), where we see the same multicomponent structure in the line $D_{3}$. This comparison allows us to suppose that the complex structure in the line D3 may be connected of additional emission components in the line.

In the work by Tuairisg et al. (2000) authors tired determination of contribution of additional emission in this line for IL CepA. For it they are subtracted a theoretical profile of the line for corresponded spectral class from the observed profile of the line. As mentioned these authors, this procedure is followed many errors: to determination of real fundamental parameters of the star, influence of the noise, model calculations etc. Main of them on our opinion are connected with determinations of the real spectral class of IL Cep A, because as mentioned in Ismailov et al.(2013) the spectral class of the star were determined by different authors is different.
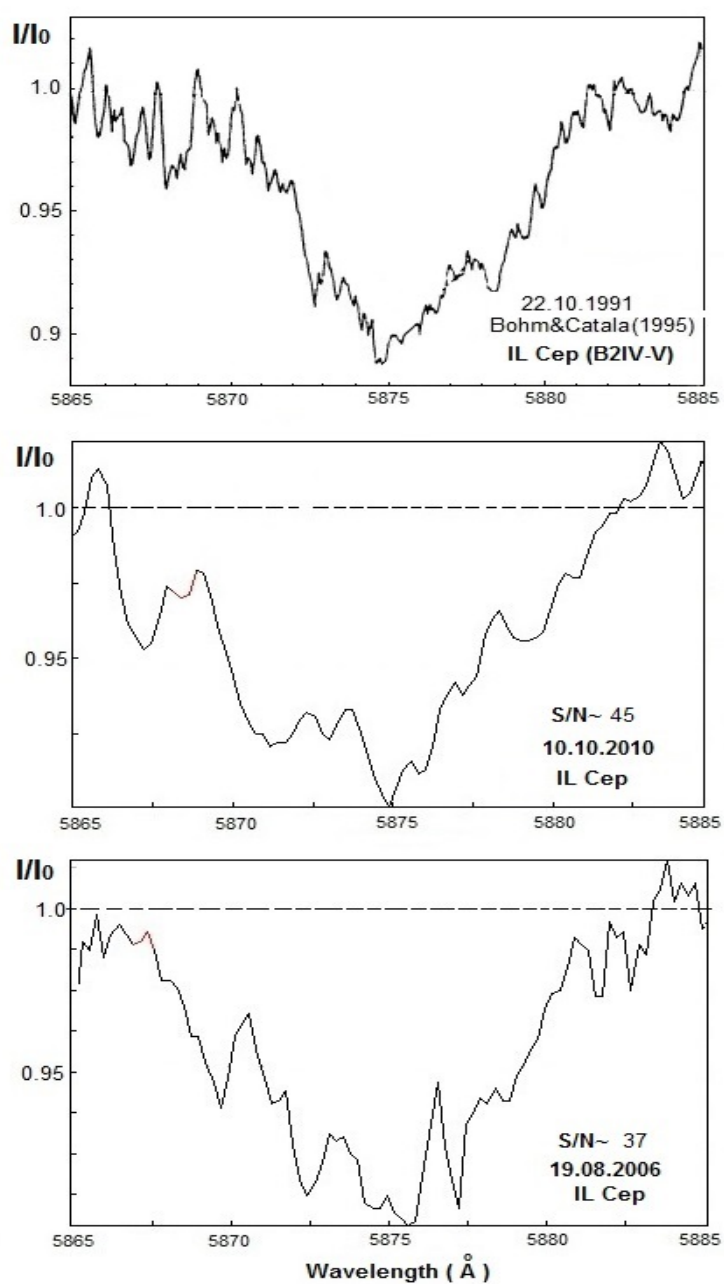

Figure 3: Profiles of the line HeI $5876 \AA$ for different stars obtained in the same equipment: on the top right panel presented a profile of the line from the work by Bohm and Catala (995). For each panel S/N level have presented. 
As a first step by using Mihalas (1964) theoretical profiles of the line HeI $5876 \AA$ (for $\mathrm{T}_{\text {eff }}=20000 \mathrm{~K}$ and $\lg g=4.0)$ and taking into account a rotational velocity of IL Cep A as $180 \mathrm{~km} / \mathrm{s}$ (Ismailov et al.2013, Melnikov et al. 1996) we have try to determine the contribution of emission component imposed to the helium absorption. In Figure $4 \mathrm{a}, \mathrm{b}$ for example we have presented a residual profiles of the line for 2007 and 2009 obtained by dividing the observational profile to the theoretical with corresponding parameters. As seen from this figures in each case we have residual emission components. This fact as a minimum shows that the observational profile of the star perhaps is consisting of superposition of emission and absorption components.
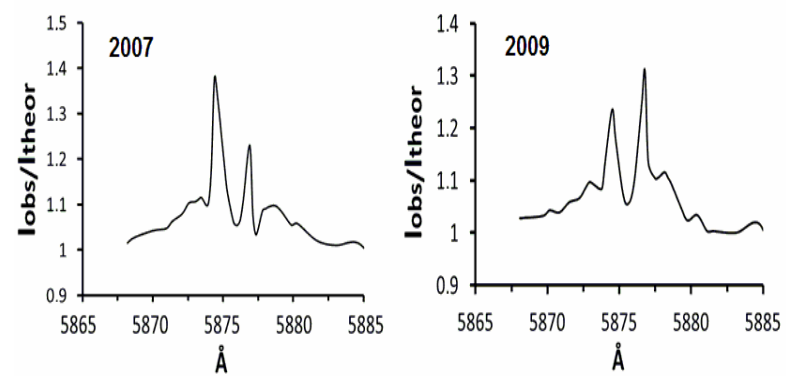

Figure 4: The residual structure obtained by dividing of the observational profile to the model.

\section{Conclusions}

Thus we have analyzed a structure of the $\mathrm{D}_{3}$ line helium in the spectrum of HAeBes IL Cep A. We concluded that the line has complex multicomponent and variable structure. Some of components in the profile are real and connected with emission from the star envelope or disk. This line represents the absorption structure which has a width on the level of the continuum about 15-18 $\AA$, and a FWHM about 6-8 A. Although equivalent widths of the line demonstrated some scatter for different seasons of observation, the mean value of this parameter is not varied from year to year.

The radial velocity in the line $\mathrm{D}_{3}$ shows the smooth variation with a minimum in 2010. This character of variation in $V r$ of the line $\mathrm{D}_{3}$ in more little scale is repeated variations of radial velocity of the emission component of $\mathrm{H} \alpha$. In 2013 and 2014 we have observed large scatter in radial velocities perhaps connected with activity in the circumstellar environment. A long time smooth variation in $V r$ perhaps occurred by dynamical motion in the binary system.

Acknowledgements. This work supported by Azerbaijan National Academy of Sciences as a priority field of scientific researches.

\section{References}

Alesian E. et al.: 2013, MNRAS, 429, 1001A.

Böhm T., Catala C.: 1995, $A \& A, \mathbf{3 0 1}, 155$.

Finkenzeller U.: 1985, $A \& A, \mathbf{1 5 1}, 340$.

Finkenzeller U., Mundt R.: 1984, $A \& A S$, 55, 109.

Galazutdinov G.A.: 1992, Preprint of the Special Astrophysical Observatory, No. 92.

Garmany C.D.: 1973, AJ, 78, 185.

Harwey J.V., Tandberg-Hanssen E.: 1968, SoPh., 3, 316. Herbig G.H.: 1960, ApJS, 4, 337.

Hill G. et al.: 1976, Publ. Dom. Astrophys. Observ., 15, 1. Hill G.: 1967, ApJSS., 14, 263.

Ismailov N.Z. et al.: 2013, Astrophys. Bull., 68, 196.

Mel'nikov S.Yu. et al.: 1996, Astron. Reports, 40, 350.

Mihalas D.M.: 1964, Pasadena, California, 220 p.

Pirzkal N. et al.: 1997, ApJ, 481, 392.

Thé P.S. et al.: 1994, $A \& A S, \mathbf{1 0 4}, 315$.

Tuairisg S.O. et al.: 2000, $A \& A S, \mathbf{1 4 2}, 225$.

Wheelwright H.E. et al.: 2010, MNRAS, 401, 1199. 